\title{
Inhaltsübersicht
}

\section{Kapitel. Elektrostatilk}

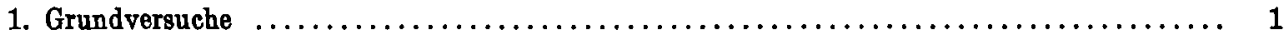

2. Die einfachsten Apparate zum Nachweis des elektrischen Zustandes $\ldots \ldots \ldots \ldots \ldots \ldots \ldots . \quad 3$

3. Gleichheit der positiven und negativen durch Reibung erzeugten Ladungen; Fluidumhypothese 5

4. Sitz der elektrischen Ladung auf einem Leiter; Flächenladungsdichte $\ldots \ldots \ldots \ldots \ldots \ldots \ldots, 7$

5. Coulombsches Gesetz; Einheit der Elektrizitätsmenge ........................ 10

6. Das elektrische Feld; elektrische Kraftlinien; elektrischer FluB; GauBscher Satz ......... 13

7. Das Potential ................................................... 18

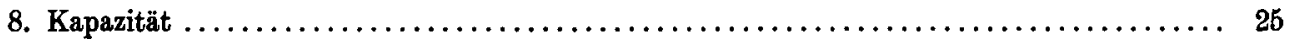

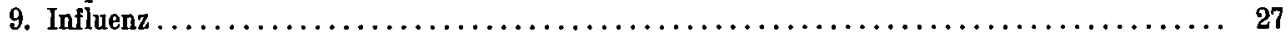

10. Anwendungen der Influenz: Doppelplatte, Potentialsonden, Elektrophor $\ldots \ldots \ldots \ldots \ldots \ldots \ldots$

11. Anwendungen der Influenz: Kondensatoren $\ldots \ldots \ldots \ldots \ldots \ldots \ldots \ldots \ldots \ldots \ldots \ldots \ldots \ldots \ldots \ldots \ldots$

12. Das elektrostatische Feld in einem Dielektrikum........................... 41

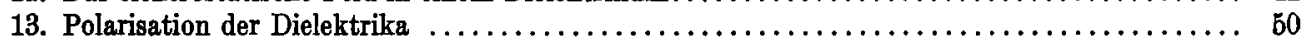

14. Die elektrische Energie; Kraftwirkungen im elektrostatischen Felde $\ldots \ldots \ldots \ldots \ldots \ldots \ldots \ldots 6$

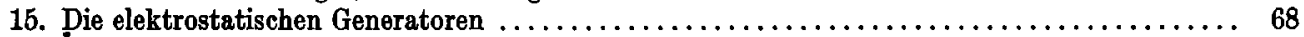

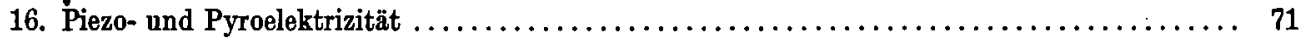

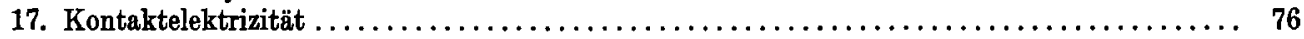

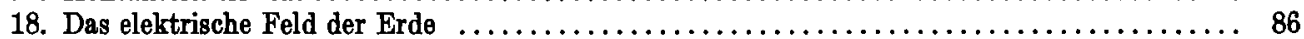

\section{Kapitel. Magnetostatik}

19. Grundtatsachen; Analogien und Differenzen zur Elektrostatik $\ldots \ldots \ldots \ldots \ldots \ldots \ldots \ldots \ldots 90$

20. Coulombsches Gesetz; magnetische Feldstärke $\ldots \ldots \ldots \ldots \ldots \ldots \ldots \ldots \ldots \ldots \ldots \ldots, 93$

21. Kraftlinien; magnetischer FluB; magnetisches Potential ..................... 96

22. Magnetstab im homogenen Magnetfeld; Messung der Feldstärke und des magnetischen Momentes 100

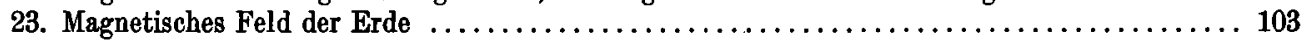

24. Einflu $B$ der Materie auf die magnetischen Erscheinungen; magnetische FluBdichte........ 107

\section{Kapitel. Stationäre elektrisehe Ströme}

25. Begriff des elektrischen Stromes; Stromstärke; Stromdichte $\ldots \ldots \ldots \ldots \ldots \ldots \ldots \ldots \ldots 122$

26. Ohmsches Gesetz ............................................ 125

27. Anwendungen des Ohmschen Gesetzes; Kirchhoffsche Sätze über Stromverzweigungen .... 141

28. Elektrische Arbeit; Stromwärme; Peltier-Effekt; chemische Umsetzungen . . . . . . . . . . 149

29. Thermoelektrizität; Peltier- und Thomson-Effekt $\ldots \ldots \ldots \ldots \ldots \ldots \ldots \ldots \ldots \ldots \ldots \ldots$

\section{Kapitel. Das elektrische und magnetische Feld stationärer Ströme}

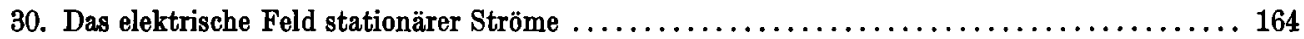

31. Oerstedscher Versuch; Magnetfeld eines geradlinigen Stromleiters $\ldots \ldots \ldots \ldots \ldots \ldots \ldots \ldots \ldots 7$

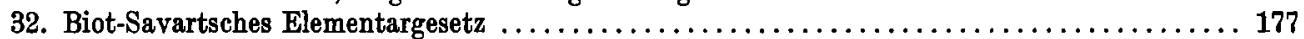

33. Äquivalenz von Strömen und Magneten; Ampères Molekularströme ................ 183

34. Die verschiedenen Maßsysteme der Elektrodynamik und ihre Beziehungen zueinander ...... 188

35. Magnetfeld von Spulen; Elektromagnete.............................. 192

36. Die Eigenschaften der ferromagnetischen Stoffe $\ldots \ldots \ldots \ldots \ldots \ldots \ldots \ldots \ldots \ldots \ldots \ldots \ldots$

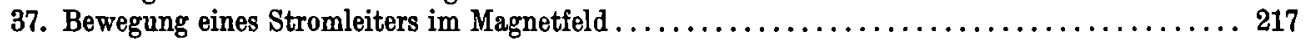

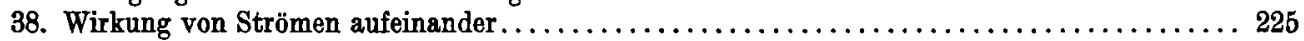

39. Der Verschiebungsstrom; Hauptgleichung des Elektromagnetismus $\ldots \ldots \ldots \ldots \ldots \ldots \ldots 230$

\section{Kapitel. Induktion}

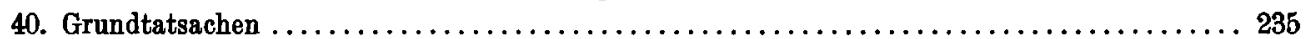

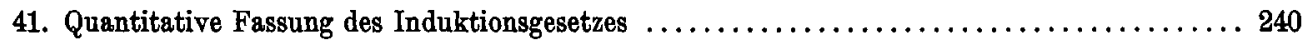


42. Einfache Anwendungen der Induktion; Erdinduktor; Messung magnetischer Felder ....... 245

43. Gegenseitige Induktion und Selbstinduktion; Anwendungen $\ldots \ldots \ldots \ldots \ldots \ldots \ldots \ldots \ldots .253$

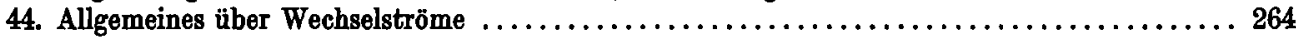

45. Wechselstromkreis mit Ohmschem Widerstand, Selbstinduktion und Kapazität.......... 271

46. Mehrphasenströme, magnetische Drehfelder $\ldots \ldots \ldots \ldots \ldots \ldots \ldots \ldots \ldots \ldots \ldots \ldots \ldots \ldots . \ldots \ldots 7$

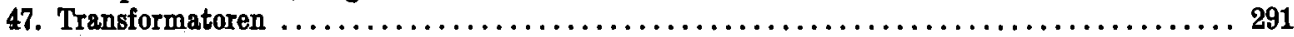

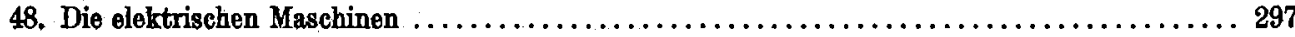

49. Die Marwellschen Gleichungen $\ldots \ldots \ldots \ldots \ldots \ldots \ldots \ldots \ldots \ldots \ldots \ldots \ldots \ldots \ldots \ldots \ldots \ldots \ldots \ldots \ldots, 312$

\section{Rapitel. Elektrisehe Schwingungen und Wellen}

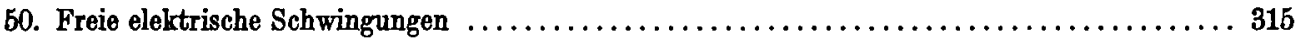

51. Erzeugung gedämpfter Schwingungen mittels der Funkenmethode $\ldots \ldots \ldots \ldots \ldots \ldots \ldots \ldots, 321$

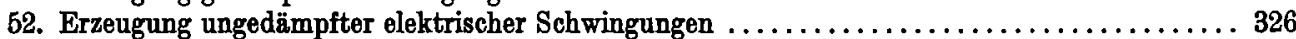

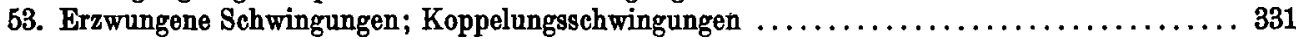

54. Ausbreitung elektrischer Wellen längs Leitungen; Telegraphengleichung $\ldots \ldots \ldots \ldots \ldots \ldots \ldots, 341$

55. Elektromagnetische Raumwellen; elektrischer Dipol und sein Strahlungsfeld . . . . . . . . 353

56. Wesensgleichheit der elektromagnetischen Wellen mit den Lichtwellen $\ldots \ldots \ldots \ldots \ldots \ldots \ldots .373$

57. Anwendung der elektrischen Wellen; Ausbreitung der Wellen um die Erde............ 378

\section{Kapitel. Elektrolyse}

68. Grundtatsachen; Mechanismus der Elektrolyse .............................. 384

59. Die Faradayschen Gesetze der Elektrolyse .............................. 390

60. Die Leitfähigkeit der Elektrolyte; Utberfühnungszahlen und Beweglichkeit von Ionen ....... 396

61. Umwandlung chemischer Energie in elektrische; Theorie der galvanischen Elemente $\ldots \ldots \ldots 408$

62. Elektrolytische Polarisation; sekundäre Elemente (Akkumulatoren) $\ldots \ldots \ldots \ldots \ldots \ldots \ldots .414$

63. Die praktischen Anwendungen der Elektrolyse $\ldots \ldots \ldots \ldots \ldots \ldots \ldots \ldots \ldots \ldots \ldots \ldots$

\section{Kapitel, Gasentledungen}

64. Die Leitfähigkeit der Gase; allgemeine Erörterungen $\ldots \ldots \ldots \ldots \ldots \ldots \ldots \ldots \ldots \ldots \ldots, 425$

65. Unselbständige Entladung bei höheren Drucken ........................... 429

66. Unselbständige Elektrizitätsleitung im Hochvakuum $\ldots \ldots \ldots \ldots \ldots \ldots \ldots \ldots \ldots \ldots \ldots \ldots$

67. Die Natur der Elektrizitätsträger im Hochvakuum $\ldots \ldots \ldots \ldots \ldots \ldots \ldots \ldots \ldots \ldots \ldots \ldots 48$

68. Anwendungen der unselbständigen Elektrizitätsleitung im Hochvakuum $\ldots \ldots \ldots \ldots \ldots \ldots 447$

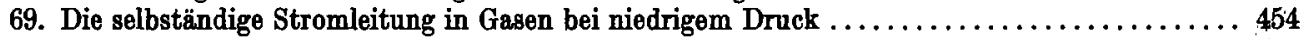

70. Die selbständige Elektrizitätsleitung in Gasen bei hohem Druck; Funken, Lichtbogen ...... 472

\section{Kapitel. Die Stromleitung in festen Körpern}

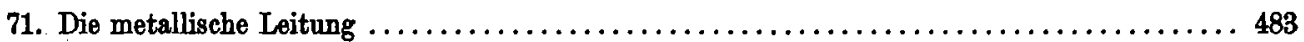

72. Die elektrische Leitung in Kristallen und Halbleitern $\ldots \ldots \ldots \ldots \ldots \ldots \ldots \ldots \ldots \ldots \ldots \ldots \ldots$

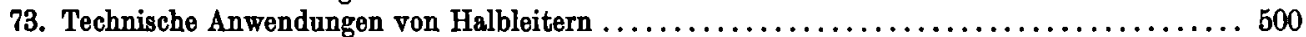

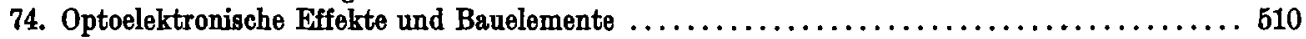

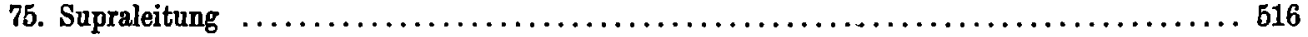

\section{Kapitel. Sondergebiete}

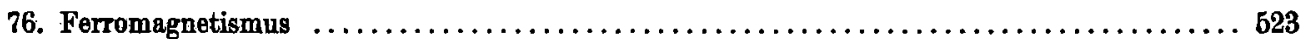

77. Antoferromagnetismus und Ferrimagnetismus $\ldots \ldots \ldots \ldots \ldots \ldots \ldots \ldots \ldots \ldots \ldots \ldots \ldots, \ldots, \ldots \ldots$

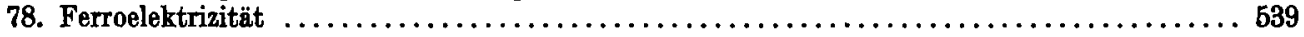

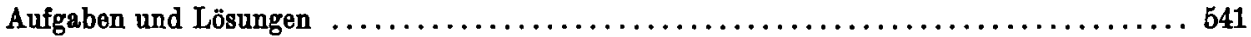

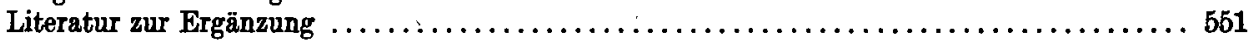

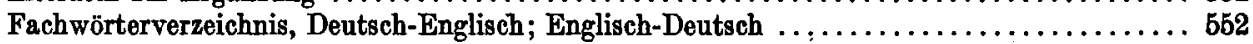

Konstanten; Energie-Einheiten; Hinweis für Zeit- und Frequenzmessungen; Verstärkungs-,

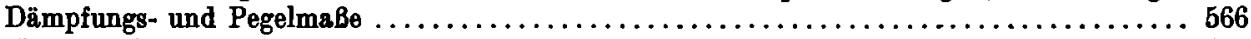

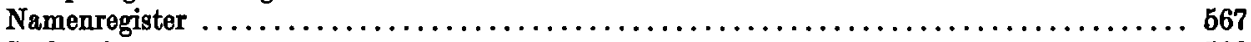

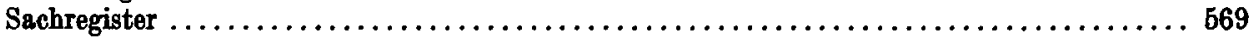

